\title{
recildunds
}

Revista Cientifica Mundo de la Investigación y el Conocimiento

Lissette Yesenia Rosero Ortega a ${ }^{\text {a }}$ Jorge Armando Rosero Aguirre ${ }^{\text {b; }}$ Elvis Rodrigo

Soledispa Cevallos ${ }^{\text {c; }}$ Omar Fabricio Sánchez Guanopatin ${ }^{\mathrm{d}}$

Utilidad de plasma rico en plaquetas en osteoartrosis de rodilla

Usefulness of platelet rich plasma in knee osteoarthritis

Revista Científica Mundo de la Investigación y el Conocimiento. Vol. 3 núm. 4., diciembre, ISSN: 2588-073X, 2019, pp. 326-339

DOI: $10.26820 /$ recimundo/3.(4).diciembre.2019.326-339

URL: http://recimundo.com/index.php/es/article/view/665

Código UNESCO: 3205 Medicina Interna

Tipo de Investigación: Artículo de Revisión

(C) RECIMUNDO; Editorial Saberes del Conocimiento, 2019

Recibido: 15/09/2019

Aceptado: 23/11/2019

Publicado: 30/12/2019

Correspondencia: lissetterosero94@gmail.com

a. Médico; Investigador Independiente; Guayaquil, Ecuador; lissetterosero94@ gmail.com

b. Especialista en Imagenología; Doctor en Medicina y Cirugía; Investigador Independiente; Guayaquil, Ecuador; jorgrosero@hotmail.com

c. Médico; Investigador Independiente; Guayaquil, Ecuador; elvisrsc09@ gmail.com

d. Médico; Investigador Independiente; Guayaquil, Ecuador; omarfabricio.sanchez@ hotmail.com 


\section{Utilidad de plasma rico en plaquetas en osteoartrosis de rodilla}

Vol. 3, núm. 4., (2019)

Lissette Yesenia Rosero Ortega; Jorge Armando Rosero Aguirre; Elvis Rodrigo Soledispa

Cevallos; Omar Fabricio Sánchez Guanopatin

\section{RESUMEN}

La osteoartrosis también conocida como osteoartritis o enfermedad articular degenerativa es una enfermedad que daña el tejido resbaladizo que cubre los extremos de los huesos de una articulación. Puede presentarse en cualquier momento de la vida y atacar cualquier articulación, pero generalmente es una enfermedad que se presenta con mayor frecuencia en la población adulta y en la articulación de la rodilla. Representa la causa más frecuente de dolor y es una de las principales causas de discapacidad y dependencia, que conlleva elevados costos en el área de la salud. El presente estudio tiene como objetivo fundamental plasmar lo relativo al uso del plasma rico en plaquetas para el tratamiento de la osteoartrosis de rodilla, específicamente su definición, mecanismos de acción, obtención, efectividad y efectos adversos. El diseño de investigación que se llevó a cabo es de tipo documental o bibliográfico. A corto plazo, las inyecciones intraarticulares de PRP son generalmente eficaces como alternativa de tratamiento en la artrosis de rodilla, en términos de alivio de dolor, función física y calidad de vida. Los riesgos están más asociados a la antisepsia con que se maneje la preparación y la aplicación, entre estos se encuentran: dolor, tumefacción post inyección, náuseas, vómitos, dolor de cabeza, gastritis y taquicardia. El uso de Plasma Rico en Plaquetas (PRP) no es nuevo, no obstante, a nivel articular es novedoso como agente regenerador. Las técnicas de obtención y las pautas utilizadas para su extracción son variadas y carecen, en muchos países, de normativas que rijan su control y garanticen su calidad. En tal sentido, resulta fundamental la práctica de ensayos experimentales y clínicos a los fines de estudiar la eficacia y seguridad de cada uno de los tipos de producto que del PRP se pueden derivar y la regulación de sus procedimientos a nivel internacional.

Palabras Claves: Plasma; Rico; Plaquetas; Osteoartrosis; Rodilla. 


\title{
Utilidad de plasma rico en plaquetas en osteoartrosis de rodilla
}

Vol. 3, núm. 4., (2019)

Lissette Yesenia Rosero Ortega; Jorge Armando Rosero Aguirre; Elvis Rodrigo Soledispa Cevallos; Omar Fabricio Sánchez Guanopatin

\begin{abstract}
Osteoarthritis also known as osteoarthritis or degenerative joint disease is a disease that damages the slippery tissue that covers the ends of the bones of a joint. It can occur at any time in life and attack any joint, but it is usually a disease that occurs most frequently in the adult population and in the knee joint. It represents the most frequent cause of pain and is one of the main causes of disability and dependence, which entails high costs in the area of health. The present study has as a fundamental objective to capture the relative use of platelet rich plasma for the treatment of knee osteoarthritis, specifically its definition, mechanisms of action, obtaining, effectiveness and adverse effects. The research design that was carried out is documentary or bibliographic. In the short term, intra-articular injections of PRP are generally effective as an alternative treatment for osteoarthritis of the knee, in terms of pain relief, physical function and quality of life. The risks are more associated with the antisepsis with which the preparation and the application are managed, among these are: pain, post injection swelling, nausea, vomiting, headache, gastritis and tachycardia. The use of Platelet Rich Plasma (PRP) is not new, however, at the articular level it is novel as a regenerating agent. The techniques of obtaining and the guidelines used for their extraction are varied and lack, in many countries, regulations that govern their control and guarantee their quality. In this sense, the practice of experimental and clinical trials is fundamental in order to study the efficacy and safety of each type of product that can be derived from the PRP and the regulation of its procedures at international level.
\end{abstract}

Keywords: Plasma; Rich; Platelets; Osteoarthrosis; Knee. 


\section{Utilidad de plasma rico en plaquetas en osteoartrosis de rodilla}

Vol. 3, núm. 4., (2019)

Lissette Yesenia Rosero Ortega; Jorge Armando Rosero Aguirre; Elvis Rodrigo Soledispa

Cevallos; Omar Fabricio Sánchez Guanopatin

\section{Introducción.}

En la actualidad la incidencia de las enfermedades degenerativas de los huesos y las articulaciones aumentan proporcionalmente a una realidad mundial: el incremento de la población de la tercera edad, siendo una de las principales la osteoartrosis (OA).

La osteoartrosis también conocida como osteoartritis o enfermedad articular degenerativa “es una enfermedad que daña el tejido resbaladizo que cubre los extremos de los huesos de una articulación. Este tejido resbaladizo se llama cartílago. Cuando esto ocurre, los huesos comienzan a rozarse entre ellos”. La fricción que produce este roce causa dolor, hinchazón y pérdida de movimiento en la articulación, incluso con el paso del tiempo padeciendo la enfermedad la articulación podría perder su aspecto natural. (Instituto Nacional de Artristis y Enfermedades Musculoesqueléticas y de la Piel de los E.E.U.U. - NIAMS, 2014)

La OA es la forma más común de artritis. Esta enfermedad puede por lo general se presenta en personas adultas o ancianos, pero no es limitante ya que puede aparecer en cualquier momento de la vida. Ataca a cualquier articulación, pero con mayor frecuencia suele afectar las manos, las rodillas, las caderas o la columna. (Enciclopedia Médica ADAM, 2018)

Para Simental, Vílchez, \& Martínez (2015) es la enfermedad crónica y degenerativa más común de las articulaciones, así como la causa más frecuente de dolor. Igualmente, representa en la población adulta, una de las principales causas de discapacidad y dependencia, que conlleva elevados costos en el área de la salud. La OA se presenta con mayor frecuencia en el sexo femenino (2:1), sin embargo, luego de la menopausia la relación hombre: mujer es 1:1. La frecuencia aumenta con la edad de la persona. "El 30\% de las personas entre 45 y 60 años de 


\section{Utilidad de plasma rico en plaquetas en osteoartrosis de rodilla}

Vol. 3, núm. 4., (2019)

Lissette Yesenia Rosero Ortega; Jorge Armando Rosero Aguirre; Elvis Rodrigo Soledispa Cevallos; Omar Fabricio Sánchez Guanopatin

edad, y más del $80 \%$ de los mayores de 80 años, presentan osteoartrosis en al menos una articulación. En todo el mundo esta enfermedad representa la cuarta causa de morbilidad en mujeres mayores de 60 años, y en hombres, la octava. (p. 353)

El tratamiento de la OA ha tenido muchos avances, entre los que se encuentra el plasma rico en plaquetas, cuya multi función de sanar, aportar factores de crecimiento y proteínas, está siendo usado en varios campos, especialmente en la OA de rodilla.

Al respecto, Moreno et al. (2015) refiere que recientemente esta herramienta terapéutica, se ha usado con mayor frecuencia y énfasis debido al empleo que se le ha dado en deportistas de élite, dada la capacidad que tiene de aumentar la regeneración ósea al ser usado conjuntamente con injertos de hueso autólogo. Por otra parte, sus beneficios de bajo coste e inocuidad, fácil manejo y su utilidad en patologías donde los tratamientos convencionales han fallado, hacen del uso del plasma rico en plaquetas más popular. Cabe destacar que sus indicaciones están en continua expansión y pueden ser aplicadas a múltiples dolencias, entre las que destacan la osteoartrosis de rodilla. (p. 131)

El presente estudio tiene como objetivo fundamental plasmar lo relativo al uso del plasma rico en plaquetas para el tratamiento de la osteoartrosis de rodilla, específicamente su definición, mecanismos de acción, obtención, efectividad y efectos adversos.

\section{Materiales y Métodos}

El uso de computadores personales con conexión a internet, fue fundamental para el desarrollo de la investigación, ya que fue por medio de este recurso que se recopiló el material 


\section{Utilidad de plasma rico en plaquetas en osteoartrosis de rodilla}

Vol. 3, núm. 4., (2019)

Lissette Yesenia Rosero Ortega; Jorge Armando Rosero Aguirre; Elvis Rodrigo Soledispa Cevallos; Omar Fabricio Sánchez Guanopatin

bibliográfico digital asociado al tema de la utilidad de plasma rico en plaquetas en la OA de rodilla, de lo cual se desprende la clasificación del presente como un diseño de investigación documental o bibliográfico.

Se usaron de diversas bases de datos, para la obtención del material bibliográfico, entre las que destacan: MedlinePlus, PubMed, Biblioteca Virtual de la Salud (BVS), SciELO, Instituto Nacional de Artritis y enfermedades musculoesqueléticas y de la piel de los Estados Unidos y Sociedad Española de Transfusión Sanguínea y Terapia Celular.

Asimismo, los descriptores que se usaron para la búsqueda fueron los siguientes: "plasma rico en plaquetas", "osteoartrosis"; "tratamiento de osteoartrosis de rodilla", "plasma rico en plaquetas en el tratamiento de la osteoartrosis de rodilla", "ventajas del plasma rico en plaquetas" y "desventajas del plasma rico en plaquetas". Estos resultados se filtraron bajo los criterios de: idioma español, relevancia, correlación temática y fecha de publicación en los últimos seis años.

\section{Resultados.}

Plasma rico en plaquetas $(P R P)$

Peris (2018) define el Plasma Rico en Plaquetas como "un volumen de plasma autólogo que contiene una concentración de plaquetas superior al nivel plasmático basal (150.000350.000/ $\mu \mathrm{L}$ ). Las plaquetas son el principal protagonista en la hemostasia y el coágulo de sangre, ya que contienen altos niveles de factores cicatrizantes". Asimismo, juega un papel importante en la reparación y regeneración de tejidos. En razón de lo anterior, es importante destacar que, si en un determinado volumen de plasma se concentran grandes cantidades de plaquetas, se puede 


\section{Utilidad de plasma rico en plaquetas en osteoartrosis de rodilla}

Vol. 3, núm. 4., (2019)

Lissette Yesenia Rosero Ortega; Jorge Armando Rosero Aguirre; Elvis Rodrigo Soledispa Cevallos; Omar Fabricio Sánchez Guanopatin

obtener un incremento exponencial de factores cicatrizantes, los cuales son fundamentales en el inicio del proceso de cicatrización y reparación de heridas. (p. 11)

\section{Mecanismo de acción}

En cuanto al mecanismo de acción del plasma rico en plaquetas Orozco Arango (2017) explica en su trabajo que existen varios mecanismos a través de los que, tanto las inyecciones intra articulares como en el hueso subcondral, influyen su efecto con una reducción del dolor articular de rodilla. Menciona además que estudios in vitro e in vivo que reportaron que:

El uso de PRP y factores de crecimiento como factor de crecimiento epidérmico, factor de crecimiento de la insulina y factor de crecimiento derivado de las plaquetas suprimen los macrófagos, fibroblastos, y los condrocitos por inhibición de la vía del $\mathrm{NFkB}$ (factor nuclear potenciador de las cadenas ligeras Kappa de las células B activadas) amortiguando y/o disminuyendo la respuesta inflamatoria a nivel sinovial y del cartílago articular. Adicionalmente, la cantidad de canabinoides endógenos presentes en el PRP actúan como ligandos de los receptores $\mathrm{CB} 1$ y $\mathrm{CB} 2$ de los condrocitos y las células sinoviales en los pacientes con OA, generando una reducción del dolor y la inflamación a través del sistema canabinoide endógeno. (p. 327) 


\section{Utilidad de plasma rico en plaquetas en osteoartrosis de rodilla}

Vol. 3, núm. 4., (2019)

Lissette Yesenia Rosero Ortega; Jorge Armando Rosero Aguirre; Elvis Rodrigo Soledispa

Cevallos; Omar Fabricio Sánchez Guanopatin

\section{Técnicas de obtención}

El PRP puede obtenerse por medio de kits desechables con "técnica cerrada" o bien, de forma manual mediante "técnica abierta". Asimismo, se pueden obtener diferentes fracciones, dependiendo del método, tales como:

- Preparado Rico en Factores de Crecimiento (PRGF),

- Plasma Rico en Plaquetas y Factores de Crecimiento (PRPGF),

- Plasma Rico en Plaquetas (PRP),

- Plasma Pobre en Plaquetas (PPP),

- Plasma Rico en Plaquetas y Rico en Leucocitos (LRPRP),

- Plasma Rico en Plaquetas y Pobre en Leucocitos (LPPRP). (Moreno et al., 2015, p. 131)

Igualmente, la Sociedad Española de Transfusión Sanguínea y Terapia Celular (2017) al respecto indica que "existen al menos 16 sistemas comerciales de separación de plaquetas, y han sido descritas diversas técnicas (diferente velocidad de centrifugación, doble centrifugación, etcétera). Dependiendo del sistema empleado, las concentraciones de plaquetas, leucocitos, eritrocitos y factores de crecimiento pueden variar". Asimismo, refiere que al usar los distintos métodos se pueden obtener diferentes fracciones, indicadas en el párrafo anterior. Por tanto, bajo la nomenclatura de PRP se pueden encerrar las diferentes fracciones obtenidas. Pese a la realización de ensayos in vitro a los fines de determinar el contenido celular y molecular de los diferentes sistemas comerciales, hasta la actualidad no se conoce la influencia que pueda tener estas diferencias en la práctica clínica. (p. 8) 


\section{Utilidad de plasma rico en plaquetas en osteoartrosis de rodilla}

Vol. 3, núm. 4., (2019)

Lissette Yesenia Rosero Ortega; Jorge Armando Rosero Aguirre; Elvis Rodrigo Soledispa Cevallos; Omar Fabricio Sánchez Guanopatin

\section{Efectividad del uso del plasma rico en plaquetas}

En una revisión realizada por Peris (2018) que incluyó 13 de los últimos ensayos clínicos aleatorizados, reclutados a partir de una búsqueda estructurada de Pubmed y Cochrane, a pesar de que no pudo concluir de manera evidente que las inyecciones intraarticulares de PRP sean efectivas como tratamiento de la osteoartrosis, mencionó los siguientes hallazgos:

A corto plazo, las inyecciones intraarticulares de PRP son generalmente eficaces como alternativa de tratamiento en la artrosis de rodilla, en términos de alivio de dolor, función física y calidad de vida. En los 12 primeros meses también existen diferencias a favor del tratamiento con PRP en la escala WOMAC, comparado con otras inyecciones, como suero salino, ácido hialurónico (AH), ozono y corticoides. En cuanto a la seguridad del PRP, podría relacionarse la aparición de inflamación y dolor local leve tras la administración del tratamiento. Sin embargo, los ensayos clínicos no arrojan datos sobre la seguridad de estas terapias a largo plazo. (p. 45)

Por otro lado, Sánchez et al. citados por Tusell Machado \& Jiménez Bodib (2018) presentaron un estudio clínico aleatorio para el tratamiento de la artrosis el cual realizaba una comparación del uso de PRP con la infiltración de AH, en el mismo lograron evidenciar que el que los pacientes tratados con PRP obtuvieron un porcentaje mayor de mejoras. Asimismo, acotan que los estudios demuestran que "este tratamiento es eficaz en las distintas fases o los 


\section{Utilidad de plasma rico en plaquetas en osteoartrosis de rodilla}

Vol. 3, núm. 4., (2019)

Lissette Yesenia Rosero Ortega; Jorge Armando Rosero Aguirre; Elvis Rodrigo Soledispa Cevallos; Omar Fabricio Sánchez Guanopatin

distintos grados de la enfermedad, lo que permite aspirar a retrasar el tratamiento definitivo de las artrosis graves, es decir, la sustitución quirúrgica de la articulación por una prótesis”. (p. 98)

Asimismo, cabe destacar que Meneses Vivar (2018) en su revisión bibliográfica concluyó que existe evidencia actual de que, en el tratamiento de la OA de rodilla, el PRP inyectado intra articularmente resulta beneficioso. En muchos de los estudios clínicos realizados hasta la actualidad se ha demostrado que el PRP conlleva en el paciente que padece la OA de rodilla a un alivio temporalmente del dolor, además de mejorar la función de la articulación afectada y, por ende, la calidad de vida de quien la padece. El PRP ha demostrado en múltiples estudios su superioridad en comparación con el ácido hialurónico (AH) y el placebo. Por último, mencionan que la liberación de los factores de crecimiento del PRP ocurre inmediatamente que se realiza el tratamiento y este puede durar alrededor de tres semanas, no obstante, el efecto clínico disminuye poco antes de un año de aplicado el tratamiento. (p. 24)

\section{Efectos adversos}

En cuanto a los efectos indeseados del tratamiento con PRP en pacientes con OA de rodilla, Pellicer García 2015, citado también por Tusell Machado \& Jiménez Bodib (2018) refiere que debido a que el PRP es una sustancia autóloga son muy bajos los riesgos y estos van a depender de la antisepsia con que se maneje la preparación y la aplicación. En cuanto a las respuestas alérgicas registradas con el uso del PRP se encuentran más relacionadas al uso de activadores como la trombina bovina, por lo que hasta la actualidad se usa muy poco. La carcinogénesis es un temor en la práctica clínica, no obstante, teniendo en cuenta "el mecanismo de acción de los factores de crecimiento sobre los receptores periféricos de membrana, se puede 


\section{Utilidad de plasma rico en plaquetas en osteoartrosis de rodilla}

Vol. 3, núm. 4., (2019)

Lissette Yesenia Rosero Ortega; Jorge Armando Rosero Aguirre; Elvis Rodrigo Soledispa Cevallos; Omar Fabricio Sánchez Guanopatin

comprender que estos no pueden modificar el ácido desoxirribonucleico, por lo que no es de esperar que tengan un efecto carcinogénico”. (p. 99)

Peris (2018) revisó un total de los 13 estudios, encontrando efectos adversos al uso del PRP solamente en 8 de estos casos. Al respecto menciona que hubo efectos secundarios específicos y no específicos, en donde, dentro del primer grupo se incluyeron el dolor y la tumefacción post-inyección, y en el segundo náuseas, síncopes, vómitos, dolor de cabeza, sudoración, gastritis y taquicardia. (p. 35)

Pilla Barroso (2017) coincide en que uno de los principales riesgos que presentan los PRP están relacionados con el sistema de activación plaquetaria mediante el empleo de trombina bovina. Asimismo, destaca la observación de anticuerpos contra los factores de coagulación $\mathrm{V}$ y IX, siendo riesgoso para el desarrollo de coagulopatías. Otras de las complicaciones o efectos adversos que pueden presentar los pacientes con el uso del PRP es la presencia de una infección, pero que está más relacionado con las infiltraciones, independientemente de la sustancia a infiltrar. En virtud de lo anterior, se ha hecho énfasis en mantener medidas rigurosas de esterilidad durante su manipulación, entre las cuales destacan la aplicación a unas pocas horas tras su extracción (4-6 horas), dado el riesgo y capacidad potencial de que se contamine la muestra y desarrolle una infección. "Otro de los posibles riesgos que pueden tener los PRP está relacionado con su capacidad sobre la quimiotaxis, proliferación celular y diferenciación tisular”. (p. 47) 


\section{Utilidad de plasma rico en plaquetas en osteoartrosis de rodilla}

Vol. 3, núm. 4., (2019)

Lissette Yesenia Rosero Ortega; Jorge Armando Rosero Aguirre; Elvis Rodrigo Soledispa

Cevallos; Omar Fabricio Sánchez Guanopatin

\section{Conclusiones.}

El uso de Plasma Rico en Plaquetas (PRP) no es nuevo, no obstante, a nivel articular es novedoso como agente regenerador. Las técnicas de obtención y las pautas utilizadas para su extracción son variadas y carecen, en muchos países, de normativas que rijan su control y garanticen su calidad. Su carácter novedoso y la variabilidad en cuanto a los procedimientos que lo rodean, aunado a la falta de control, conlleva a un nivel de evidencia científica bajo.

Las ventajas de este tratamiento a corto plazo, son generalmente eficaces como alternativa de tratamiento en la artrosis de rodilla, en términos de alivio de dolor, función física y calidad de vida. No obstante, existen riesgos los cuales se encuentran asociados principalmente a la antisepsia con que se maneje la preparación y la aplicación, entre los efectos adversos se encuentran: dolor, tumefacción post inyección, náuseas, vómitos, dolor de cabeza, gastritis y taquicardia. Asimismo, se encuentran asociados al uso de activadores como la trombina bovina, resultando respuestas alérgicas. Otro riesgo está representado por el posible desarrollo de coagulopatías dada la observación de anticuerpos de factores de coagulación.

La composición del PRP podría alterar sus propiedades biológicas de manera importante.

En consecuencia, la gran variabilidad de técnicas de preparación de PRP podría conllevar a ciertos resultados clínicos indeseados, lo que puede poner en duda la eficacia de este tratamiento. En tal sentido, resulta fundamental la práctica de ensayos experimentales y clínicos a los fines de estudiar la eficacia y seguridad de cada uno de los tipos de producto que del PRP se pueden derivar y la regulación de sus procedimientos a nivel internacional. 


\section{Utilidad de plasma rico en plaquetas en osteoartrosis de rodilla}

Vol. 3, núm. 4., (2019)

Lissette Yesenia Rosero Ortega; Jorge Armando Rosero Aguirre; Elvis Rodrigo Soledispa

Cevallos; Omar Fabricio Sánchez Guanopatin

\section{Bibliografía.}

Enciclopedia Médica ADAM. (30 de Noviembre de 2018). MedlinePlus. Recuperado el 10 de Diciembre de 2019, de https://medlineplus.gov/spanish/osteoarthritis.html

Instituto Nacional de Artristis y Enfermedades Musculoesqueléticas y de la Piel de los E.E.U.U. - NIAMS. (nOVOEMBRE de 2014). NIAMS. Recuperado el 18 de Diciembre de 2019, de https://www.niams.nih.gov/es/informacion-de-salud/osteoartritis

Meneses Vivar, E. (2018). EFICACIA DEL PLASMA ENRIQUECIDO EN PLAQUETAS EN EL TRATAMIENTO DE LA OSTEOARTRITIS DE RODILLA. Tesis de Grado, UNIVERSIDAD DE ALICANTE, Facultad Ciencias de la Salud. Recuperado el 20 de Diciembre de 2019, de https://rua.ua.es/dspace/bitstream/10045/76458/1/EFICACIA_DEL_PLASMA_ENRIQU ECIDO_EN_PLAQUETAS_E_MENESES_VIVAR_ERICKA_YESSENIA.pdf

Moreno, R., Gaspar, M., Jiménez, J., Alonso, J. M., Villimar, A., \& López, P. (2015). Técnicas de obtención del plasma rico en plaquetas y su empleo en terapéutica osteoinductora. Farmacia Hospitalaria, 39(3), 130-136. Recuperado el 19 de Diciembre de 2019, de http://scielo.isciii.es/pdf/fh/v39n3/02articuloespecial01.pdf

Orozco Arango, J. A. (2017). Terapias intervencionistas para manejo de dolor en osteoartrosis de rodilla sintomática. Revista de la Sociedad Española del Dolor, 24(6), 324-332. Recuperado el 21 de Diciembre de 2019, de http://scielo.isciii.es/pdf/dolor/v24n6/11348046-dolor-24-06-00324.pdf

Peris, X. (2018). Plasma Rico en Plaqueta y Artrosis de Rodilla. Tesis de Grado, Universitat Jaume I, Facultad de Ciescias de la Salud, Castellón. Recuperado el 18 de Diciembre de 2019 , http://repositori.uji.es/xmlui/bitstream/handle/10234/176565/TFG_2018_PerisFuertesXav ier.pdf?sequence $=1 \&$ isAllowed $=y$

Pilla Barroso, J. (2017). "BENEFICIOS DEL TRATAMIENTO CON INFILTRACIÓN DE PLASMA RICO EN PLAQUETAS EN PACIENTES CON OSTEOARTRITIS EN LA CONSULTA EXTERNA DE TRAUMATOLOGÍA DEL HOSPITAL ALFREDO NOBOA MONTENEGRO DURANTE EL PERÍODO ENERO - JUNIO DEL 2017”. Tesis de Grado, UNIVERSIDAD REGIONAL AUTÓNOMA DE LOS ANDES, Facultad de Ciencias Médicas, Ambato. Recuperado el 10 de Diciembre de 2019, de http://dspace.uniandes.edu.ec/bitstream/123456789/6530/1/PIUAMED068-2017.pdf

Simental, A., Vílchez, J. F., \& Martínez, H. G. (2015). El plasma rico en plaquetas en osteoartrosis de rodilla: una alternativa de tratamiento. Cirugía y Cirujanos, 83(4), 352358. Recuperado el 05 de Diciembre de 2019, de https://www.elsevier.es/es-revistacirugia-cirujanos-139-pdf-S0009741115001000 


\section{Utilidad de plasma rico en plaquetas en osteoartrosis de rodilla}

Vol. 3, núm. 4., (2019)

Lissette Yesenia Rosero Ortega; Jorge Armando Rosero Aguirre; Elvis Rodrigo Soledispa

Cevallos; Omar Fabricio Sánchez Guanopatin

Sociedad Española de Transfusión Sanguínea y Terapia Celular. (24 de Mayo de 2017). Informe de la Agencia Española de Medicamentos y Productos Sanitarios sobre el uso de Plasma Rico en Plaquetas. Boletín de la Sociedad Española de Transfusión Sanguínea y Terapia Celular, 17(2), 1-40. Recuperado el 22 de Diciembre de 2019

Tusell Machado, O., \& Jiménez Bodib, J. (2018). Concentrado de plaquetas para el tratamiento de la artrosis de rodilla. Acta Médica del Centro, 12(1), 93-103. Recuperado el 19 de Diciembre de 2019, de https://www.medigraphic.com/pdfs/medicadelcentro/mec2018/mec1811.pdf

$$
\text { (C) }(\mathcal{Q} \otimes(0)
$$

RECONOCIMIENTO-NOCOMERCIAL-COMPARTIRIGUAL

CC BY-NC-SA

ESTA LICENCIA PERMITE A OTROS ENTREMEZCLAR, AJUSTAR Y CONSTRUIR A PARTIR DE SU OBRA CON FINES NO COMERCIALES, SIEMPRE Y CUANDO LE RECONOZCAN LA AUTORÍA Y SUS NUEVAS CREACIONES ESTÉN BAJO UNA LICENCIA CON LOS MISMOS TÉRMINOS. 